Didáctica. Lengua y literatura

ISSN-e: 1988-2548

https://dx.doi.org/10.5209/dida.65936

\title{
El método comparativo funcional: una vía para enseñar gramática
}

\author{
Kenia María Velázquez Avila'; Ernan Santiesteban Naranjo²
}

Recibido: 8 de mayo de 2017 / Aceptado: 4 de septiembre de 2019

Resumen. El comunicador social debe poseer una competencia comunicativa, sin embargo, mediante la aplicación de métodos de la investigación se constató que los estudiantes presentan deficiencias que limitan los procesos de comunicación profesional. Una de las causas que genera esta contradicción es la insuficiente argumentación de los métodos didácticos. Para corroborar lo antes mencionado, se realizó un estudio diacrónico del proceso de enseñanza-aprendizaje de la Gramática, y a partir de las limitaciones encontradas en las teorías didácticas se propone el método comparativo funcional. Se describen, igualmente, cada una de las acciones realizadas durante el período experimental. Es significativo señalar que los resultados que se presentan son parte del proyecto de investigación: La enseñanza de la Gramática en la Educación Superior, de la Universidad de Las Tunas, Cuba. Dicho proyecto está asociado a la Red Iberoamericana de Pedagogía en la que se pretende implementar el método propuesto en diversas universidades de América Latina.

Palabras clave: enseñanza-aprendizaje, gramática española, método comparativo funcional, comunicador social.

\section{[en] The functional comparative method: a way to teach grammar}

\begin{abstract}
The social communicator must possess a communicative competence; however, through the application of research methods, it was found that they present inadequacies that limit the processes of professional communication. One of the causes that generates this contradiction is the insufficient argumentation of the didactic methods. In order to corroborate the above, a diachronic study of the teaching-learning process of Grammar was carried out, and from the limitations found in didactic theories the functional comparative method is proposed. It also describes each of the actions carried out during the experimental period. It is significant to note that the results presented are part of the research project: The Teaching of Grammar in Higher Education, University of Las Tunas, Cuba. This project is associated to the Ibero-American Network of Pedagogy in which it is tried to implement the proposed method in several universities of Latin America.
\end{abstract}

Keywords: teaching-learning, Spanish grammar, functional comparative method, social Communicator.

\section{[fr] Méthode fonctionnel comparatif pour une façon grammaire enseigner}

Résumé. Le journaliste doit avoir une compétence communicative, cependant, en appliquant les méthodes de recherche se sont avérés avoir des lacunes qui limitent les processus de communication

1 Centro de Estudios de Pedagogía Universidad de Las Tunas, Cuba keniamariavelazquezavila@gmail.com

2 Editorial Académica Universitaria Universidad de Las Tunas, Cuba esantiesteban2012@gmail.com 
professionnelle. L'une des causes qui ont généré cette contradiction est un argument insuffisant des méthodes didácticos. Para corroborer ce qui précède, il serealizó une étude diachronique de la grammaire enseignement et d'apprentissage, et des limitations rencontrées dans les théories didactiques propose la méthode comparative fonctionnelle. Est décrit également chacune des actions prises au cours de la période experimental. Es important de noter que les résultats présentés font partie du projet de recherche: enseignement de la grammaire dans l'enseignement supérieur, l'Université de Las Tunas, Cuba. Le projet est associé au Réseau latino-américain de l'éducation où elle a l'intention de mettre en œuvre les universités proposées dans la méthode Amérique latine.

Mots-clés: l'enseignement et l'apprentissage, grammaire espagnole, méthode comparative fonctionnelle, Communicateur social

Sumario: 1. Introducción. 2. El proceso de enseñanza-aprendizaje de la gramática española en la carrera Comunicación Social. 3. Método comparativo funcional: una propuesta para desarrollar el aprendizaje de la gramática. 4. Los procedimientos del método comparativo funcional. 5. Discusión de los resultados. 6. Conclusiones. Bibliografía.

Cómo citar: Velázquez Avila, K. M.; Santiesteban Naranjo, E. (2019). El método comparativo funcional: una vía para enseñar gramática, Didáctica. Lengua y literatura, 31, 29-45.

\section{Introducción}

El Modelo del Profesional de la carrera Comunicación Social plantea la formación de un profesional con clara conciencia de su papel en la sociedad, con la convicción del sustancial aporte que puede lograr la comunicación en el desarrollo social; debe ser capaz de gestionar la comunicación con profesionalismo, desde su posición de mediador, comprometido con sus públicos, su profesión y su país.

De lo expuesto se infiere que el comunicador social debe constituir un modelo lingüístico para la sociedad. Por tanto, es necesario que conozca y utilice de forma adecuada la lengua materna, lo que requiere del domino de las estructuras gramaticales (clases léxico-gramaticales y unidades sintácticas).

La gramática ocupa un lugar destacado en el sistema lingüístico. El conocimiento gramatical es de significativa importancia en la comunicación, ya que los procesos de codificación y decodificación de significados se dificultan cuando aparece un error en la sintaxis.

Por consiguiente, en el Plan de Estudio de la referida carrera se ubica como disciplina de formación general Lengua Española y Cultura. Esta disciplina tiene como finalidad proporcionarle al estudiante los conocimientos y habilidades comunicativas para su futuro desempeño profesional. Entre los objetivos formulados en dicho programa está el desarrollo de los fundamentos necesarios para la apropiación por parte del estudiante de las formas adecuadas de expresión de la lengua materna, tanto oral como escrita.

En concordancia con lo planteado, entre las asignaturas se ubica Gramática Española que tiene declarado como uno de los objetivos esenciales del programa el empleo adecuado de las estructuras gramaticales del idioma español en la redacción de textos de carácter profesional. Sin embargo la experiencia acumulada como profesores investigadores miembros de dos proyectos relacionados con la enseñanza de la gramática y la aplicación de diferentes tipos de evaluaciones durante el proceso de enseñanzaaprendizaje permitieron constatar que los estudiantes presentan insuficiencias en: 
- los conocimientos sobre los criterios semántico, sintáctico y pragmático de las estructuras gramaticales en los textos profesionales;

- el desarrollo de las habilidades de análisis gramatical en los textos profesionales; $y$

- el uso de las estructuras gramaticales a partir de los valores de la sociedad cubana.

En lo anterior se revela una contradicción entre las exigencias del Modelo del Profesional que requieren la formación de un profesional capaz de comunicarse en sus contextos de actuación y la realidad que evidencia el insuficiente desarrollo de la competencia gramatical de los estudiantes, que limita el proceso de comunicación. Ello presupone dirigir el proceso de enseñanza-aprendizaje de la Gramática Española hacia la caracterización estilística de las estructuras, en correspondencia con las particularidades de los estilos.

\section{El proceso de enseñanza-aprendizaje de la gramática española en la carrera Comunicación Social}

Los antecedentes de la formación del comunicador social en Cuba están vinculados al desarrollo de la publicidad y las relaciones públicas en el país. Desde 1959, se inició un proyecto que entró en antagonismo con las formas de propiedad capitalista existentes, con el objetivo de analizar el consumo y los distintos modos de propiciarlo.

Los medios masivos fueron contemplados dentro del proceso de nacionalización que abarcó todos los sectores de la economía cubana, por lo que se integraron al control del Estado bajo estructuras propias de financiamiento, con un rediseño paulatino de sus formas y contenidos. Asimismo, la Escuela Profesional de Publicidad dejó de existir en 1960. Posteriormente, diferentes organismos y entidades permitieron la actualización de los conocimientos sobre las tareas de propaganda, aunque no funcionaron como centros docentes directamente vinculados a la publicidad.

Con la caída del campo socialista (1990), fue necesario buscar otras fuentes de ingreso para la economía cubana. Como consecuencia de la situación anterior se decidió sustituir (1991) la carrera de Periodismo por la de Comunicación Social, y se creó la Facultad de Comunicación Social en la Universidad de La Habana. Esta carrera, desde sus inicios, ubicó la asignatura Gramática Española como parte de su Plan de Estudio, debido a la importancia que tiene el contenido gramatical para la formación del comunicador social.

El estudio histórico-lógico del referido proceso se efectuó sobre la base de las modificaciones realizadas en el proceso de enseñanza-aprendizaje de la Gramática Española, a partir del desarrollo alcanzado en las teorías didáctica y lingüística. Los aspectos seleccionados para realizar el estudio son: los enfoques determinados para la enseñanza de la Gramática Española y la relación de la gramática con otras ciencias lingüísticas en el proceso de enseñanza-aprendizaje. Dichos aspectos permitieron realizar un estudio diacrónico de la concepción de los objetivos, el sistema de contenidos y los métodos didácticos.

De los aspectos anteriores se determinaron tres etapas. Primera: la enseñanza de la Gramática Española desde una concepción oracional (1991-1999). Segunda: 
la enseñanza de la Gramática Española desde una concepción textual (2000-2009). Tercera: la enseñanza de la Gramática Española desde una concepción discursiva (2010-actualidad).

\section{Primera etapa: La enseñanza de la Gramática Española desde una concepción oracional (1991-1999)}

El análisis del Plan de Estudio permitió constatar que se orientó hacia un perfil amplio del comunicador social. La revisión del programa de la asignatura y de las orientaciones metodológicas adjuntas permitió comprobar que el enfoque propuesto para el proceso de enseñanza-aprendizaje fue el descriptivo, que se centró en el análisis metagramatical.

Los objetivos declarados estaban dirigidos hacia el análisis de las estructuras gramaticales desde la oración, es decir, el estudio de la gramática por la gramática y no en función de la comunicación. El sistema de contenidos se organizó a partir de la propuesta de Porro (1977 y 1978). Uno de los principales aportes de estos autores fue el establecer tres criterios para el estudio de las estructuras gramaticales.

Los criterios fueron el semántico, el formal y el funcional. Ellos, aun con su carácter inmanente, permitieron profundizar en el estudio y en el conocimiento de las reglas gramaticales, elementos indispensables para desarrollar una competencia gramatical, sin embargo, no se consideraron ni los significados contextuales, ni las posibilidades de uso que pueden revelar dichas estructuras en y para la comunicación.

El método propuesto para la enseñanza-aprendizaje de la gramática fue el descriptivo. Su objetivo era establecer las relaciones existentes entre los distintos elementos oracionales. Consistió en delimitar los componentes del sintagma predicativo y, posteriormente, aislar los elementos que integran el sintagma nominal sujeto y el sintagma verbal, para describir su estructura.

Se hizo énfasis en la memorización de definiciones y clasificaciones, lo que resultó esquemático y formal. Se partió de una concepción inmanente del fenómeno lingüístico, sin considerar los avances de la lingüística del texto.

La relación de la gramática con otras ciencias lingüísticas desde el proceso de enseñanza-aprendizaje fue nula. En el caso particular de la estilística, la relación no era posible -según Roméu (2007)-; la causa radicó en que las gramáticas tradicionales nunca se ocuparon de los estilos funcionales. Se explica esta omisión porque el carácter restringido de su análisis impidió que pudiera revelarse el estilo de un mensaje.

Lo antes descrito permite afirmar que durante la primera etapa, el proceso de enseñanza-aprendizaje de la Gramática Española se concibió de forma disciplinar, centrado en el análisis metagramatical. El enfoque propuesto para la enseñanza gramatical fue el descriptivo, basado en el análisis de la forma, la función y el significado de "las partes de la oración". Esta concepción limitó la relación interdisciplinaria de la gramática con la estilística y el desarrollo de la competencia gramatical del comunicador social.

Segunda etapa: La enseñanza de la Gramática Española desde una concepción textual (2000-2009)

El análisis del Plan de Estudio permitió constatar que se orientó hacia un perfil de comunicación institucional. En él se establecieron con mayor precisión las particula- 
ridades que demandaron la formación del comunicador social con rasgos diferenciadores del perfil periodístico, y ello representó un aporte en cuanto al reconocimiento social y la legitimación del ejercicio profesional en este campo.

La revisión del programa de la asignatura y de las orientaciones metodológicas adjuntas permitió constatar que el enfoque propuesto para el proceso de enseñanzaaprendizaje fue el comunicativo que se centró en el proceso comunicativo, y las estrategias para lograrlo.

Este enfoque promovió la exigencia de rebasar el límite de la oración y dirigió el estudio de las estructuras gramaticales hacia las relaciones de significado entre las oraciones en los textos, al considerar que hay propiedades gramaticales más allá de la frontera de la oración. Esto propició la relación interdisciplinaria entre la gramática y la semántica, no así con la estilística, ya que los estilos funcionales constituyen variaciones de matices estilísticos y no de significados.

Los objetivos declarados estuvieron centrados en el análisis de las estructuras gramaticales desde el texto.

El sistema de contenidos se organizó sobre la base del estudio de las estructuras gramaticales desde el texto, y se propuso desde de la concepción aportada por Grass (2002). Esta autora planteó desarrollar en los estudiantes las habilidades de análisis en los textos científicos, literarios, jurídicos, coloquiales, y publicitarios. Para alcanzar la referida meta, Grass propone extender a los textos de las restantes tipologías, la metodología para el análisis y comentario de los textos literarios.

La implementación de estos fundamentos en el proceso de enseñanza-aprendizaje de la Gramática Española posibilitó la interdisciplinariedad de los conocimientos gramaticales. El análisis de las estructuras gramaticales se realizó vinculado con los significados (semántica), pero no con la estilística. Por un lado, la extrapolación de una metodología para textos literarios no permite profundizar en las características específicas de los distintos estilos. Por otro lado, la metodología fue diseñada para el análisis integral del texto, que incluye el contenido gramatical, pero no lo prioriza.

Para la caracterización se asumieron los mismos criterios aportados por Porro (1977 y 1978). El método propuesto esencialmente para el análisis fue el gramatical, que tuvo como objetivo identificar y caracterizar las estructuras gramaticales en el texto. Dicho método superó al descriptivo, al reconocer el texto como la unidad lingüística donde las estructuras gramaticales adquieren su verdadero significado, y facilitó su análisis en correspondencia con su contexto verbal, pero no consideró los contextos extra-verbales.

Lo antes descrito permite afirmar que durante la segunda etapa, el proceso de enseñanza-aprendizaje de la Gramática Española se centró en el análisis de las estructuras gramaticales vinculado con los significados. El enfoque propuesto para la enseñanza gramatical fue el comunicativo, basado en el análisis de las estructuras gramaticales desde el texto. Esta concepción permitió establecer relaciones interdisciplinarias de la gramática con la semántica, pero limitó la relación con la estilística y por ende, el desarrollo de la competencia gramatical del comunicador social.

\section{Tercera etapa: La enseñanza de la Gramática Española desde una concepción discursiva (2010-actualidad)}

El análisis del Plan de Estudio permitió constatar que se orienta hacia la formación del comunicador social con un perfil amplio que integra los diversos ámbitos en 
los que se manifiesta la comunicación, tales como los institucionales, académicos y mediáticos. Posteriormente, la revisión del programa de la asignatura y de las orientaciones metodológicas adjuntas permitió constatar que el enfoque propuesto para el proceso de enseñanza-aprendizaje es el descriptivo comunicativo funcional que se centra en la relación del discurso con la gramática.

Por enfoque descriptivo comunicativo funcional se entiende aquel proceso de reflexión metalingüística que permite descubrir la funcionalidad de las estructuras gramaticales en relación con lo que significa el texto, la intención y el contexto en que significa, en esencia, propone la apropiación de la funcionalidad textual de las estructuras gramaticales a partir del discurso.

Dicha teoría posibilita establecer una relación entre la competencia metalingüística y la competencia lingüística. La primera se inscribe en los procesos metacognitivos, los cuales consisten en la reflexión y el control sobre aquellos del propio pensamiento, como resultado de una conciencia creciente acerca de la naturaleza de las funciones cognitivas. Su finalidad es la reflexión sobre las posibilidades que ofrece el código lingüístico para la comunicación en diversas situaciones comunicativas (Rodríguez, 2018). Por tanto, el desarrollo de la competencia metalingüística garantiza el desarrollo de la competencia lingüística.

Los elementos anteriores han condicionado que los objetivos didácticos declarados estén centrados en el análisis de las estructuras gramaticales desde el discurso. Consecuentemente, el sistema de contenidos está dirigido hacia la integración entre los procesos cognitivos, la interacción y las estructuras sociales, elementos que permiten abordar el estudio del lenguaje en el ámbito social.

Esta concepción plantea -según la teoría aportada por Toledo (2011) - un tránsito de la gramática del texto hacia la gramática del discurso. Se propone, además, el estudio de las estructuras gramaticales desde los criterios semántico, sintáctico y pragmático. La caracterización semántica incluye el estudio de las variaciones de sentido/significado que pueda portar una estructura gramatical según la intención y finalidad comunicativas del emisor. Lo anterior prioriza el sentido/significado contextual al significado lógico-primario.

La caracterización sintáctica abarca el estudio de la composición morfológica y la función sintáctica de las estructuras gramaticales. La caracterización pragmática, por su parte, comprende el estudio de la función de las estructuras gramaticales en relación con la situación de comunicación: circunstancias de lugar, tiempo, jerarquía del receptor y tipos de textos. En este criterio se propone como un elemento más a considerar los estilos funcionales de la lengua.

Esta nueva concepción propicia de forma acertada la interdisciplinariedad de la gramática, la semántica y la pragmática, sin embargo, no siempre permite profundizar en su relación con la estilística. Esto se debe a que la caracterización pragmática de las estructuras gramaticales en un texto de determinado estilo funcional de la lengua, no necesariamente implica la caracterización estilística de dichas estructuras.

Según Fernández (1983, p. 144): “Conocido el Renacimiento español (...) no se explica a Garcilaso. Pudo haber sido un versificador eficaz, como Boscán. Pero fue un gran poeta. En vano buscaríamos en su época razones para ello. La época no nos explica de él sino lo que él comparte con una gran familia de renacentistas, de alta y baja calidad".

Es imprescindible abordar el conocimiento acerca de los contextos en los cuales se inscribe el texto. La situación comunicativa aporta lo general, lo que pueden com- 
partir los textos. La estilística, por su parte, destaca lo que el contexto no puede explicar, la cualidad única de las estructuras gramaticales, la irrepetible, la individual.

Al respecto, Fernández (1983, p. 21) planteó: “(...) la estilística es, en efecto, aquella que más cerca está, no del contexto, sino del texto mismo; que-sin desdeñar el estudio de todas las circunstancias colaterales- se propone el afrontamiento de la obra en sí, el último esfuerzo por hacerla asequible”. Estas consideraciones sustentan la pretensión de atomizar el criterio estilístico del pragmático.

El método propuesto esencialmente para el análisis gramatical fue el discursivo funcional o descriptivo comunicativo funcional, que tiene dos objetivos, el descriptivo y el explicativo. El primero se basa en la abundancia de recursos gramaticales que poseen las lenguas para expresar una misma idea y de ahí se analiza la forma en que los hablantes los seleccionan.

El segundo objetivo está orientado a investigar las causas que originan que las lenguas posean tales recursos, así como las causas de la estructuración de los discursos (la información conocida generalmente va al principio y no al final). Las explicaciones que proporciona este método son gramaticales, cognitivas y sociales o interactivas.

Uno de los principales aportes del empleo de este método es que no se recurre a ejemplos conformados, alejados de la realidad, sino que selecciona las estructuras de la propia realidad comunicativa, y no se limita solo al texto, también considera los contextos lingüístico, extra-lingüístico y etnográfico en que se emite; sin embargo, este método fue extrapolado de las ciencias lingüísticas a la didáctica de la lengua sin la contextualización requerida. Por tanto, no contempla aspectos esenciales que se manifiestan en el proceso de enseñanza-aprendizaje, como son los procedimientos didácticos.

Lo antes descrito permite afirmar que durante la tercera etapa, el proceso de enseñanza-aprendizaje de la Gramática Española se centró en el análisis de las estructuras gramaticales desde el discurso. El enfoque propuesto para la enseñanza gramatical fue el descriptivo comunicativo funcional, que considera los contextos lingüístico, extra-lingüístico y etnográfico. Esta concepción permitió establecer relaciones interdisciplinarias de la gramática con la semántica y la pragmática, pero aún es insuficiente la relación con la estilística y, por ende, el desarrollo de la competencia gramatical del comunicador social.

Del estudio realizado en cada una de las etapas analizadas, se identificaron dos tendencias.

- De un enfoque descriptivo se transita a un enfoque comunicativo y posteriormente al descriptivo comunicativo funcional, que considera los contextos lingüístico, extra-lingüístico y etnográfico, sin embargo, los métodos y los procedimientos determinados por estos enfoques no siempre permiten profundizar en la caracterización estilística de las estructuras gramaticales en los textos profesionales.

- De una concepción disciplinar de la gramática, basada en el análisis descriptivo de la forma, la función y el significado de "las partes de la oración", se transita hacia una concepción interdisciplinaria que incluye elementos semánticos, y posteriormente pragmáticos, sin embargo es insuficiente aún la relación que se establece con la estilística, a partir de los contextos profesionales. 


\section{Método comparativo funcional: una propuesta para desarrollar el aprendizaje de la gramática}

Una adecuada selección de los métodos didácticos permite la organización interna del proceso de enseñanza-aprendizaje de la Gramática Española. En este sentido, la competencia gramatical puede desarrollarse mediante los métodos de la didáctica general, pero los métodos particulares, específicos para la enseñanza del contenido gramatical, permiten organizar la actividad de aprendizaje de los estudiantes de un modo más eficiente, al considerar las particularidades del sistema de conocimientos, habilidades y valores.

A partir de las limitaciones reveladas en el estudio histórico realizado se ofrece el método comparativo funcional cumple una función organizadora. La función organizadora establece que los estudiantes tomen conciencia de los procedimientos, se apropien del método mediante la sistematización, y logren la asimilación de los conocimientos y las habilidades, desde sus propias soluciones, criterios o valoraciones para alcanzar los objetivos propuestos. El método que se propone permite al profesor utilizar procedimientos para el seguimiento del diagnóstico, y al estudiante, ejercer el autocontrol de sus conocimientos y habilidades, que facilitan evaluar sistemáticamente el desarrollo de la competencia gramatical como proceso y resultado.

En el proceso de enseñanza-aprendizaje de la Gramática Española, el método comparativo funcional establece relaciones con el objetivo y el contenido. El objetivo se refleja no solo en el programa de la asignatura, sino también en el estudiante, quien debe hacerlo suyo. El contenido se expresa en el texto, y el método en el propio estudiante que lo exterioriza en habilidades para el análisis gramatical del contenido que se revela en el texto, y así cumple con el objetivo propuesto.

Para alcanzar el objetivo, el profesor dirige al estudiante en la resolución de los problemas que se plantean, pero a medida que el estudiante se apropia de la esencia que contiene el objetivo lo enriquece y transforma. El objetivo y el contenido del texto determinan qué métodos se deben emplear.

El objetivo está dirigido a la redacción de textos profesionales en los que se empleen de forma adecuada las estructuras gramaticales. El estudiante debe dominar como contenido las características semánticas, sintácticas, pragmáticas y estilísticas de las estructuras gramaticales en los textos científicos, periodísticos y publicitarios. Ello indica la necesidad de proponer un nuevo método para el estudio del contenido gramatical.

El método de referencia posibilita el desarrollo de la competencia gramatical como configuración psicológica, de manera que en la medida que esta se internalice el resto de las subcompetencias comunicativas también lo harán. Tal es el caso de la competencia discursiva que implica el dominio de estrategias textuales en las cuales se combinan las estructuras gramaticales, los significados y los contextos para la redacción de diferentes tipos de textos.

La aplicación del método comparativo funcional ofrece una lógica, en la que el estudiante de la carrera Comunicación Social debe partir de la caracterización semántica, sintáctica, pragmática y estilística de las estructuras gramaticales. Posteriormente debe actuar en consecuencia con lo aprendido y transformarse en un agente socializador competente, en correspondencia con la sociedad cubana.

La comparación es la confrontación de objetos con el fin de poner de manifiesto los rasgos de semejanza o de diferencia entre ellos (o unos y otros a la vez). Es una 
premisa importante de la generalización. Desempeña una gran función en los razonamientos por analogía. Los juicios que expresan el resultado de la comparación sirven para descubrir el contenido de los conceptos acerca de los objetos.

El término comparativo determina la confrontación de una misma estructura gramatical desde perspectivas diferentes con el fin de poner de manifiesto los rasgos de semejanza o de diferencia existentes. El término funcional subraya la semejanza con el método discursivo funcional, debido a que ambos se centran en las funciones de las estructuras gramaticales. La diferencia radica en que a través del método propuesto el estudiante determina las características esenciales de las referidas estructuras, mediante la comparación de dos textos de diferentes estilos funcionales. Su objetivo, además, no se reduce a describir y explicar, como es el caso del método discursivo funcional.

El método comparativo funcional se contextualiza en el proceso de enseñanzaaprendizaje de la Gramática Española, tiene como objetivo demostrar el empleo adecuado de las estructuras gramaticales en los textos profesionales, a partir de la determinación de las semejanzas y diferencias de la funcionalidad de una misma estructura gramatical en diferentes estilos. Este método se caracteriza porque el profesor organiza la participación de los estudiantes de forma activa y consciente a través de un sistema de interrogantes, a través de las cuales el estudiante gradualmente compara las estructuras objeto de estudio en los diferentes textos y mediante una reflexión metalingüística y determina las particularidades en correspondencia con sus características semánticas, sintácticas, pragmáticas y estilísticas.

Este método está compuesto por dos componentes: el textual y el intertextual. El componente textual expresa el estudio de las estructuras gramaticales desde el texto como unidad lingüística del discurso, ya que este conforma el nivel superior del estudio gramatical y constituye el nivel de partida y de llegada del análisis gramatical.

El texto posee criterios de textualidad que lo caracterizan, entre los que se encuentra la coherencia formal que está determinada por las estructuras gramaticales, lo que se evidencia en el plano de la expresión, en la conformación sintáctica. Es importante significar, además, la función social y cultural que desempeñan los textos (como unidades del discurso) en el desarrollo de la personalidad del estudiante. El concebir los contextos pragmáticos de producción de significados, el estilo individual del emisor, así como los contextos psicológicos del receptor y emisor, propician que la estructura gramatical analizada sea portadora de emociones, sentimientos y valores, cuya esencia desarrolla la competencia gramatical desde la socialización durante el proceso de enseñanza-aprendizaje.

La relación sujeto-sujeto se revela al abordar el trabajo metodológico con los textos. Ello considera los roles del emisor y receptor en el proceso de sescodificación y codificación de la significación textual. En este sentido, la propuesta proporciona al estudiante nuevos elementos que le permiten analizar la variedad temático-referencial establecida por el emisor, así como los valores estilístico-gramaticales del texto, a la vez que posibilita el desarrollo de habilidades para el análisis de los códigos culturales, ideológicos, éticos, psicológicos. Por tanto, favorece las relaciones interpersonales, así como la influencia del ser social sobre la conciencia individual y la función determinante de esta última en la construcción o apropiación de la cultura.

Un texto no es un elemento autónomo, sino que está constituido por elementos de otros textos. Cada texto es inherentemente intertextual. Por esta razón, el segundo componente es el intertextual que expresa la relación que se establece entre los 
textos científico, periodístico y publicitario, los contextos profesionales en los que se desempeña el comunicador social y las estructuras gramaticales.

Esto representa que durante el proceso de análisis interdisciplinario de las estructuras gramaticales, los estudiantes deben compartir no solo conocimientos gramaticales y convenciones que regulan el uso del lenguaje, sino también conocimientos sobre la concepción del mundo. Dicho componente posibilita establecer conexiones entre el texto y las representaciones de conocimiento previo compartidas por los estudiantes como miembros de una comunidad, lo que les permite comprender el mundo exterior y percibir la coherencia del texto desde las vivencias y la cultura.

El método comparativo funcional se basa en la comparación de dos textos de diferentes estilos funcionales de la lengua, que posean una misma clave semántica. Esta concatenación asegura el conocimiento previo del estudiante y el nivel profundo de análisis, que se concreta en la redacción de un tercer texto. Lo anterior permite establecer las relaciones entre el signo y su referente, entre el signo, sus usuarios emisor-receptor y sus contextos de utilización, así como entre los signos y hacia el interior de ellos mismos.

El método comparativo funcional se fundamenta desde la unidad de lo instructivo con lo educativo. Es instructivo porque constituye una vía de acercamiento al conocimiento científico y posibilita la asimilación de estrategias metacognitivas al potenciar el aprender a aprender. Permite la sistematización de los conocimientos gramaticales, el establecimiento de los nexos lógicos entre conceptos, principios y regularidades del proceso estudiado, la profundidad de los conocimientos interdisciplinarios al jerarquizar las vías encaminadas a propiciar la solución de los ejercicios, y la ampliación del campo de conocimiento de los estudiantes, al lograr que ellos se adentren en la investigación de problemas profesionales.

Es educativo porque permite que el estudiante desarrolle su pensamiento creador y forme una concepción científica del mundo, basada en los principios y leyes de la pedagogía cubana. Potencia el fortalecimiento de la relación entre los conocimientos interdisciplinarios y la necesidad de profundizar en la práctica. Asimismo, favorece el trabajo en grupo, la atención a las individualidades, las relaciones estudiantescolectivo y profesores-estudiantes, el respeto a la opinión ajena, el desarrollo de estilos adecuados de discusión y sentimientos de empatía.

La desventaja del método comparativo funcional radica, esencialmente en el tiempo que se requiere para su empleo porque presupone la comparación de dos textos, para luego proceder a la producción de un tercero. Las ventajas de su uso son las siguientes:

- provee a los estudiantes de un modelo para el análisis de la funcionalidad de las estructuras gramaticales que permite redactar textos en diferentes estilos;

- propicia que los estudiantes resuelvan por sí mismos nuevas tareas cognoscitivas, lo que presupone dar respuestas a múltiples problemas profesionales (comunicarse de forma adecuada en los diferentes contextos)

- permite a los profesores demostrar las relaciones causa-efecto, o sea, revelar los elementos contextuales que determinan las variaciones en los estilos y, por consiguiente, las características que deben revelar las estructuras gramaticales en correspondencia con la situación comunicativa en que se empleen

- facilita a los profesores crear nuevas situaciones de aprendizaje que desarrollen los diferentes estados dinámicos, desde la sistematización de emociones, 
sentimientos y vivencias, que propician el desarrollo de la competencia gramatical y

- posibilita, además, la sistematización de los conocimientos de diversas ciencias: gramática, semántica, pragmática y estilística.

De la relación dialéctica entre los componentes textual e intertextual surge el carácter comunicativo del método comparativo funcional, que propicia la contextualización del contenido gramatical en función de los procesos de comunicación profesional. Ello constituye el elemento esencial para ubicar la gramática en función de la comunicación.

En la instrumentación de esta concepción didáctica se debe tener presente que en la actividad independiente el estudiante debe recibir con claridad las orientaciones que precisa para el desarrollo de los ejercicios, para que, en la medida que se desarrolle su competencia gramatical, requiera de menos ayuda. Resulta imprescindible asegurar la atención a las diferencias individuales, de forma que se pueda motivar el desarrollo de aquellos estudiantes que no alcanzan el nivel de logros deseados, así como la atención que demanden los que muestran un rendimiento superior.

\section{Los procedimientos del método comparativo funcional}

Una organización metodológica adecuada del proceso de enseñanza-aprendizaje de la Gramática Española en la carrera Comunicación Social posibilita que los estudiantes asimilen los conceptos, leyes, principios y teorías, así como, las habilidades y los valores declarados en el programa de la asignatura. Asimismo, la integración de los conocimientos gramaticales con los estilísticos, propicia que desde esta perspectiva, los estudiantes se desempeñen en sus contextos profesionales y que cumplan con los objetivos propuestos en el Modelo del Profesional.

Los procedimientos constituyen la base instrumental para desplegar el método. Son los que permiten llevar a la práctica, la lógica articulada del método. La aplicación de los procedimientos contribuye a organizar el contenido que el estudiante debe asimilar, propicia la reflexión y comprensión consciente del conocimiento, favorece en el estudiante la valoración de dicho conocimiento y de su propio aprendizaje, motiva la búsqueda de causas y argumentos, desarrolla el pensamiento, y permite la asimilación de un modelo a seguir para el logro de su aprendizaje.

El método comparativo funcional está conformado por cinco procedimientos que contienen las tareas docentes que realizan tanto los estudiantes como el profesor durante el proceso de enseñanza-aprendizaje. A continuación se abordan cada uno de ellos.

\section{Selección de los textos para comparar}

En este primer procedimiento se inicia con la selección de los textos para comparar. El estudiante desempeña un rol protagónico durante el proceso de enseñanza-aprendizaje de la Gramática Española, al seleccionar conjuntamente con el profesor los textos que se van a comparar. Los textos deben estar vinculados al contexto profesional del comunicador social, y se deben emplear de forma sistemática durante el proceso de enseñanza-aprendizaje, lo que posibilita evaluar el desarrollo de la com- 
petencia gramatical desde la integración de saberes en correspondencia con la esfera de actuación del comunicador social.

Los textos seleccionados deben ser asequibles, responder a las necesidades sociales de los estudiantes, expresar el mismo contenido (clave semántica) y estar en diferentes estilos funcionales de la lengua, ser portadores de elementos de carácter cognitivo y afectivo, de forma tal que prepare al estudiante para la vida. Deben estar acorde con la posición ideológica que aspira a formar la sociedad cubana en sus miembros, y poseer el mismo nivel de complejidad en cuanto a su estructura. Es primordial que en ambos textos seleccionados se emplee la misma estructura gramatical que constituye objeto de estudio.

Los textos deben partir de la propia realidad comunicativa y ser provenientes de diversas fuentes, tanto en soporte plano como digital. Esta diversidad posibilita acceder a bibliografía actualizada de diferentes países, esencialmente en el caso de los textos periodísticos y publicitarios que les ofrecen a los estudiantes experiencias cognitivas. Dichos textos facilitan la sistematización de los conocimientos, el desarrollo de las habilidades, la potenciación del trabajo en grupo y la dinamización de la comunicación interpersonal para intercambiar ideas, sentimientos y opiniones. Estos elementos responden a la interrogante ¿qué requisitos deben tener los textos?

\section{Presentación de los textos}

En este procedimiento se debe hacer énfasis en los aspectos etimológicos de interés. Los estudiantes, bajo la dirección del profesor, proceden al análisis de la situación comunicativa. Se deben analizar las referencias históricas, geográficas, bibliográficas y culturales de ambos textos, el medio de difusión empleado, sus características, el público al que se dirige, el tema desarrollado, las incógnitas léxicas, los sentidos contextuales, la intención y finalidad de los autores al codificar cada texto. Se deben clasificar los textos según los estilos funcionales de la lengua, caracterizar cada uno de ellos y demostrar cómo se evidencia cada característica en el texto. Se concluye con la comparación de ambos textos.

Estos elementos responden a las interrogantes ¿qué dice?, ¿por qué lo dice?, ¿para qué lo dice?, ¿qué importancia tiene lo que dice?, ¿quién lo dice?, ¿dónde lo dice?, ¿cuándo lo dice?, ¿a quiénes se lo dice?, ¿cómo lo dice? y ¿qué características tiene lo que dice?

\section{Determinación de la funcionalidad de la estructura gramatical (objeto de estudio) en ambos textos}

En este procedimiento el profesor debe diseñar ejercicios que permitan la caracterización semántica, sintáctica y pragmática de la estructura gramatical objeto de estudio (en correspondencia con el programa de la asignatura). El estudiante, por su parte, debe realizar la búsqueda, la exploración reflexiva de conocimientos, debe acceder al análisis de las partes constitutivas de las estructuras gramaticales que estudia, analizar y caracterizar cada uno de los elementos que integran los criterios constituidos, así como su identificación y clasificación. Es indispensable alcanzar la visión integral del texto, establecer relaciones interdisciplinarias, buscar argumentos, defenderlos, hacer valoraciones y reevaluar el estudio. Se concluye con la comparación de las estructuras gramaticales de cada texto. 
Este procedimiento responde a las interrogantes: ¿qué significado porta la estructura gramatical estudiada?, ¿cómo está estructurada?, ¿cómo se escribe?, ¿cómo funciona? y ¿por qué se emplea esta y no otra?

\section{Caracterización estilística de la estructura gramatical (objeto de estudio) en} ambos textos

En este procedimiento el profesor debe diseñar ejercicios que permitan la caracterización estilística de la estructura gramatical objeto de estudio. En dichos ejercicios se deben establecer los aspectos a comparar sobre la información que se brinda en los textos. Las preguntas deben propiciar la determinación de las semejanzas y diferencias entre una misma estructura gramatical en ambos textos, para lo cual se requiere encontrar las características generales de cada estructura gramatical, las particulares a un estilo funcional de la lengua, y las singulares, pertenecientes al estilo individual del autor.

Este procedimiento posibilita la participación activa del estudiante para analizar y llegar a conclusiones. Dichas exigencias están dirigidas a promover la reflexión, la formación de generalizaciones teóricas y de juicios valorativos sobre los conocimientos y las habilidades que se aprenden, lo que propicia el desarrollo del pensamiento del estudiante y, con ello, el desarrollo de la competencia gramatical. Lo anterior presupone arribar a conclusiones que permiten aplicar el contenido asimilado durante la clase en otras situaciones comunicativas.

Los elementos abordados responden a las interrogantes: ¿qué características estilísticas posee la estructura gramatical estudiada?, ¿por qué se empleó de esta forma y no otra? y ¿qué sucede si se cambia por otra?

\section{Contextualización de las estructuras gramaticales en diversas situaciones comunicativas}

En este procedimiento, el profesor presenta un problema profesional en un contexto diferente a los anteriores. Para darle solución, el estudiante debe redactar un tercer texto en un estilo diferente a los dos con los que ha trabajado, y ser consecuente con el tema abordado. De esta forma, mediante un análisis metalingüístico, emplea la estructura gramatical objeto de estudio. Se concluye con la revisión del texto, que enfatiza en las características que portan las estructuras gramaticales objeto de estudio.

\section{Discusión de los resultados}

Para validar la propuesta se empleó un experimento pedagógico formativo. El método experimental permitió no solo conocer el citado proceso como actividad pedagógica, sino que además estableció las bases para la rendición de su ulterior comportamiento.

El experimento empleado es de tipo sucesional proyectado y, de acuerdo con el grado de control de variables, se clasifica como pre-experimento. El diseño aplicado es pre-experimental con pre-test y post-test. Se desarrolló en el contexto natural de la Universidad de Las Tunas. El experimento se concibió con carácter transformador, verificador y de evaluación cualitativa. Para la realización del experimento, se planificaron cinco acciones. 


\section{Primera acción: seleccionar el grupo experimental}

Para la comprobación práctica de la hipótesis, se seleccionó una muestra que coincide con la población (los 27 estudiantes que conforman el primer año de la carrera Comunicación Social). Se determinó el primer año, porque es en este donde se imparte la asignatura Gramática Española. De este modo, las variables de tipo grupal (la auto-elección, la mortalidad y la maduración) fueron neutralizadas. Las variables auto-elección y maduración no incidieron, ya que los estudiantes fueron asignados al grupo por los estándares establecidos y el tiempo de experimentación fue corto. En el caso de la mortalidad, no se produjeron bajas.

Segunda acción: determinar el nivel de desarrollo de la competencia gramatical de los sujetos en su estado inicial (prueba de entrada).

Para la evaluación de la competencia gramatical se confeccionó una prueba pedagógica. La prueba se aplicó en la segunda semana del curso. Para ello se controlaron las condiciones siguientes: los mismos evaluadores, a la misma hora, el mismo día de la semana, el mismo local y la misma atmósfera afectiva antes, durante y después de las pruebas realizadas, con el fin de controlar variables de tipo ambientales (naturales y artificiales).

La prueba pedagógica aplicada se caracterizó, además, por tener dos textos de diferentes estilos funcionales de la lengua y una misma clave semántica. Las estructuras gramaticales empleadas en ambos textos eran equivalentes, lo que posibilitó su comparación. El referido instrumento concluyó con la redacción de otro texto en un estilo funcional diferente a los anteriores. En la redacción, los estudiantes emplearon las mismas estructuras gramaticales comparadas.

Para evaluar la variable se empleó el procedimiento de la estadística descriptiva, que permite categorizar la variable dependiente en intervalos establecidos (escalas ordinales asociadas a escalas de intervalos). Se emplearon estas escalas ordinales, ya que son las adecuadas para variables cualitativas como la competencia gramatical durante un proceso de enseñanza-aprendizaje.

Las categorías empleadas fueron: muy alto, alto, medio, bajo y muy bajo, en una gradación desde un nivel superior (cuatro) hacia un nivel inferior (cero). Para categorizar la variable se consideraron las valoraciones de las tres dimensiones, con sus tres indicadores y las escalas valorativas correspondientes.

Los resultados obtenidos en la aplicación de la prueba de entrada corroboraron que el desarrollo de la competencia gramatical de los estudiantes de primer año de Comunicación Social se encontraba en un nivel bajo.

Durante la aplicación de la prueba de entrada se constató que los estudiantes reproducen de forma memorística las características generales de las estructuras gramaticales, o sea, conocen los criterios semánticos, formales y funcionales preestablecidos para cada estructura gramatical, desde una concepción estructuralista, sin embargo no conocen los elementos pragmáticos y estilísticos que pueden portar dichas estructuras en correspondencia con las diversas situaciones comunicativas. Estas insuficiencias incidieron de forma general en la evaluación de la dimensión saber, que fue ubicada en un nivel medio.

La dimensión saber hacer fue ubicada en un nivel bajo (uno). Estos resultados se deben a que los estudiantes no identifican según los cuatro criterios establecidos, las estructuras gramaticales en los textos seleccionados, las mayores insuficiencias se en- 
contraron en la determinación de la funcionalidad de dichas estructuras en correspondencia con los estilos. Los estudiantes recurren a la estructura del texto para describir y explicar la distribución de las estructuras gramaticales, por lo que se realiza un análisis que se acerca más al estructuralismo de Skinner que a los aportes de la lingüística del texto. Asimismo, se constataron deficiencias en la redacción de textos profesionales, ya que las situaciones comunicativas son más dinámicas, diversas y contradictorias que la teoría, por esa razón es inevitable tener un dominio pleno de las posibilidades de uso de cada estructura para poder emplearla en diversos contextos profesionales.

La dimensión saber ser también se encuentra en el nivel bajo (uno); esto se debe a que los estudiantes reconocen el sistema de valores que deben poseer, pero no los demuestran desde los valores estilísticos de las estructuras gramaticales, elemento que constituye la esencia de su función como agentes socializadores.

Para determinar la calidad de la competencia gramatical en el grupo experimental, se empleó la prueba de pares igualados y rangos no-paramétricos del Wilcoxom. Para ello se tomó el nivel representativo de la prueba de un 0,05 de fallo. Los datos se procesaron en un paquete estadístico SPSS. La referida prueba se realizó sobre la base de dos hipótesis:

- Hi: existen diferencias significativas entre la constatación inicial y final del grupo experimental.

- Ho: no existen diferencias significativas entre la constatación inicial y final del grupo experimental.

La prueba de pares igualados y rangos no-paramétricos del Wilcoxom corrobora los resultados revelados anteriormente.

\section{Tercera acción: implementar los procedimientos del método comparativo funcional}

Para la implementación de los procedimientos se emplearon los 90 minutos del horario de continuidad en la sesión de la mañana durante dos días de la semana (segundos turnos de los martes y miércoles), y se realizó de forma sistemática durante el primer semestre del curso escolar 2015-2016. El control de las condiciones del experimento consistió, fundamentalmente, en la no-introducción de otras variables didácticas con efectos en el campo de acción de las dimensiones e indicadores durante el período experimental. El análisis del equilibrio entre las características de los participantes (experimental) y la verificación de las evaluaciones fueron centralizadas en un investigador principal.

Para la implementación de la propuesta se elaboraron diez guías de ejercicios. Las referidas guías partieron de la comparación de dos textos de diferentes estilos funcionales. Dicha comparación posibilita determinar las particularidades de las características de las estructuras gramaticales, en correspondencia con el estilo funcional del texto, aspecto que propicia la intertextualidad, y por consiguiente el trabajo con el texto como unidad lingüística del discurso.

\section{Cuarta acción: analizar las particularidades de la aplicación de la propuesta}

Para el desarrollo del experimento se confeccionó una guía para el registro de datos con el objetivo fundamental de conocer cómo se iba desarrollando la organización 
general del experimento. El registro puso énfasis en las particularidades durante la implementación de los procedimientos del método comparativo funcional, o sea, la organización general del proceso, especialmente en la evaluación, en el cumplimiento de los objetivos por clases, las valoraciones sobre las guías de ejercicios, las emociones, los sentimientos de los estudiantes por los resultados obtenidos, las opiniones y sugerencias, entre otros aspectos.

Quinta acción: determinar los efectos sobre el grupo experimental, en correspondencia con los objetivos propuestos (prueba de salida)

Para el análisis de los resultados obtenidos se aplicó una prueba de salida en la última semana del semestre. La prueba de salida aplicada se caracterizó por tener dos textos de diferentes estilos funcionales de la lengua, con una misma clave semántica e iguales estructuras gramaticales, dichos textos fueron objeto de comparación por los estudiantes. El referido instrumento concluyó con la redacción de un tercer texto en otro estilo funcional, en el que debían aplicar las estructuras gramaticales estudiadas.

Por cuanto, este instrumento presenta idénticas características a la prueba de entrada, con el propósito de neutralizar las variables de medidas (el efecto práctico, reactivo e inestabilidad de medidas), se realizaron dos pruebas diferentes, pero equivalentes en la forma y el contenido. Esta acción concluye con las valoraciones generales del experimento que se abordan en el siguiente epígrafe.

La prueba de salida realizada al grupo que conforma la muestra, y el análisis del registro de datos del experimento sustentan la afirmación anterior, ya que la competencia gramatical de los estudiantes de primer año de la carrera Comunicación Social se ubicó en un nivel alto.

La prueba de pares igualados y rangos no paramétricos del Wilcoxom demuestra la alta significación de la propuesta para el desarrollo de la competencia gramatical de los estudiantes de primer año de la carrera Comunicación Social. La aplicación de la prueba estadística antes referida aportó los siguientes resultados: es altamente significativo. Este grado de significación está dado por la influencia del proceso de enseñanza-aprendizaje y por los procedimientos del método comparativo funcional. El resultado alcanzado demuestra la factibilidad de la propuesta, y con ello se rechaza la hipótesis nula y se adopta la de investigación. Lo anterior conduce a la conclusión de que el método comparativo funcional es propicio para desarrollar la competencia gramatical.

En sentido general, la propuesta permitió aplicar a nuevas situaciones lo aprendido, aportar sus puntos de vista, argumentar, expresar suposiciones, generar hipótesis, según las exigencias de cada texto. De esa forma, el análisis realizado potenció el saber, el saber hacer y el saber ser, lo que propició el desarrollo de la competencia gramatical y, por consiguiente, un desempeño gramatical en los diferentes contextos profesionales.

\section{Conclusiones}

- El proceso de enseñanza-aprendizaje evidencia de manera general, como tendencia, el tránsito de una gramática tradicional basada en el análisis metagramatical de la oración hacia un análisis interdisciplinario del texto como unidad 
lingüística del discurso, sin embargo, se limita la relación con la estilística, lo que evidencia una carencia epistemológica que justifica el surgimiento del método comparativo funcional.

- Los procedimientos propuestos permiten desplegar el método comparativo funcional y posibilitan la asimilación del contenido gramatical en su interrelación con los elementos semánticos, pragmáticos y estilísticos.

- En la comprobación experimental quedó demostrado que los procedimientos del método comparativo funcional desarrollan la competencia gramatical de los estudiantes de la carrera Comunicación Social.

\section{Bibliografía}

Fernández, Roberto. (1983): Idea de la estilística. La Habana, Pueblo y Educación.

Grass, Élida. (2002): Textos y abordajes. La Habana, Pueblo y Educación.

Porro, Migdalia. (1977): Forma, función y significado de las partes de la oración. La Habana, Libros para la Educación.

Porro, Migdalia. (1978): Sintaxis del español contemporáneo. La Habana, Libros para la Educación.

Rodríguez, Carmen (2018): Actividad metalingüistica y reflexión gramatical. [ref. de 14 de septiembre de 2019]. Disponible en Web: https://getxolinguae2013.wordpress. com/2013/03/25/actividad-metalinguistica-y-reflexion-gramatical-carmen-rodriguezgonzalo/

Romeu, Angelina. (2007): El enfoque cognitivo, comunicativo y sociocultural en la enseñanza de la lengua y la literatura. La Habana, Pueblo y Educación.

Toledo, Alicia. (2011): Gramática española contemporánea: de la gramática de la lengua a la gramática del discurso. La Habana, Pueblo y Educación. 